\title{
A Case of Huge Follicular Adenomatoid Odontogenic Tumor Treated by Fenestration and Delayed Enucleation
}

\author{
Nishio T, Kobashi H*, Matsukawa M, Tanaka S and Kogo M \\ First Department of Oral and Maxillofacial Surgery, Japan \\ *Corresponding author: Hironobu Kobashi, First Department of Oral and Maxillofacial Surgery, Japan
}

\section{ARTICLE INFO}

Received: 幽 February 12, 2019

Published: March 06, 2019

Citation: Nishio T, Kobashi H, Matsukawa M, Tanaka S, Kogo M. A Case of Huge Follicular Adenomatoid Odontogenic Tumor Treated by Fenestration and Delayed Enucleation. Biomed J Sci \& Tech Res 15(3)-2019. BJSTR. MS.ID.002719.

Keywords: Adenomatoid Odontogenic Tumor (AOT); Enucleation; Fenestration; Follicular type

\begin{abstract}
Adenomatoid odontogenic tumors are typically located either centrally in the jaw, peripherally in the soft tissue overlying tooth bearing areas, or around an impacted maxillary canine. We encountered a 26-year-old woman with a facial deformity and lesion that extended into the maxillary, which was treated by fenestration and delayed enucleation. The patient initially presented with swelling of her left cheek that was confirmed to be associated with a tumor by panoramic X-ray and computed tomography. The lesion was well-defined an radiolucent, had a small calcific mass, and included an impacted tooth in the left maxillary sinus. Biopsy and fenestration were performed under local anesthesia, and a pathological diagnosis of follicular-type adenomatoid odontogenic tumor was made. The size of the lesion had reduced by 6 months after the fenestration and biopsy. Therefore, we performed enucleation of the tumor and extraction of the impacted tooth under general anesthesia. The patient remained free of symptoms and recurrence at 6 months after surgery.
\end{abstract}

\section{Introduction}

Adenomatoid odontogenic tumor (AOT), first described by Stafne in 1948 [1], is a rare and benign epithelial tumor that most commonly occurs in the anterior maxillary region (especially in the canine region) of younger women [2]. There are three types of AOT: follicular, which has a central lesion associated with an impacted tooth; extrafollicular, which has no relation with an impacted tooth; and peripheral [3]. The follicular type is often associated with the crown and part of the root of an impacted tooth, typically affecting the maxillary canines but rarely affecting the permanent molars [4]. Radiographically, a cystic lesion containing teeth and a calcified body may be present, while histologically, the tumor cells appear as duct- or corolla-like structures with calcified material [5]. In this report, we describe a rare case of a huge follicular-type AOT that extended into the maxillary sinus of a young woman.

\section{Case Report}

A 26-year-old woman presented to our department with swelling of the left cheek for several months. There was no history of trauma, discharge, or any altered sensation. Intraorally, she complained of pain from the left upper central incisor to the left upper first premolar, and examination revealed that the buccal and palatal gingiva overlying this area was swollen. However, the swelling was non-tender to palpation and had a firm consistency. Also, the left upper canine was not present. Extra-orally, the swelling was diffuse and extended on the left cheek. Panoramic $\mathrm{X}$-ray examination revealed a radiolucent area with an impacted left upper lateral incisor extending into the left maxillary sinus (Figure 1A). The roots of the left upper median incisor and canine were separate, but the roots of left upper premolars and first molar had been absorbed. Subsequent contrast-enhanced computed tomography (Figures 1B \& 1C) revealed the presence of a lesion measuring $40 \times 40 \times 36 \mathrm{~mm}$. Many radiopacities were found within the radiolucent lesion, including calcified material and impacted tooth. The lesion extended to the nasal cavity and maxillary sinus, the buccal cortical bone was thinning, and the palatine bone had been absorbed.

Biopsy was performed under local anesthesia in an outpatient clinic. Serous liquid, calcified material, and a thick fibrous capsule were encountered after perforating the labial cortical plate, and a jelly-like specimen was collected that had a cystic appearance. At this time, we therefore performed a fenestration to reduce the tumor size, stimulate bone renewal, and improve the chance of a 
favorable cosmetic outcome. A histologic diagnosis of folliculartype AOT was made from the biopsy sample. Changes in the lesion were monitored by follow-up CT, and a reduction in the tumor size with accompanying bone renewal were confirmed about 6 months after the fenestration procedure (Figures 1D \& 1E). Therefore, we performed enucleation of the maxillary tumor and apicoectomy of the left upper canine under general anesthesia (Figure 2A). The tumor was approached intraorally via a reflected mucoperiosteal flap extending from the right upper lateral incisor to the left upper first molar. To remove bone from around the fenestration, the firm mass was separated from the adjoining bone along its margin and removed with the involved tooth (left upper lateral incisor).

Apicoectomy was also performed for the involved canine, but no other teeth were associated with the lesion. Hemostasis was achieved, and primary closure was completed with absorbable sutures. The total size of the enucleated tissue, including the impacted tooth, was about $35 \mathrm{~mm}$ and had a thick cyst-like coating (Figures 2A \& 2B). As at biopsy, the contents of the enucleated tumor had a jelly-like consistency. The histopathological report of the enucleated lesion confirmed the diagnosis of follicular-type AOT. Ova-l and polygon-shaped cells were present with ductal and corolla-like structures (Figures 2C \& 2D). A mass of calcifications was also present. At 1 year after the final surgery, the left cheek swelling, and the facial asymmetry had greatly improved (Figure 3). Bone renewal was also confirmed and the deformity of the bone of the front wall of the maxillary sinus and the alveolar bone of the buccal side had improved. Of note, there had been no surgical site infection or clinical recurrence.

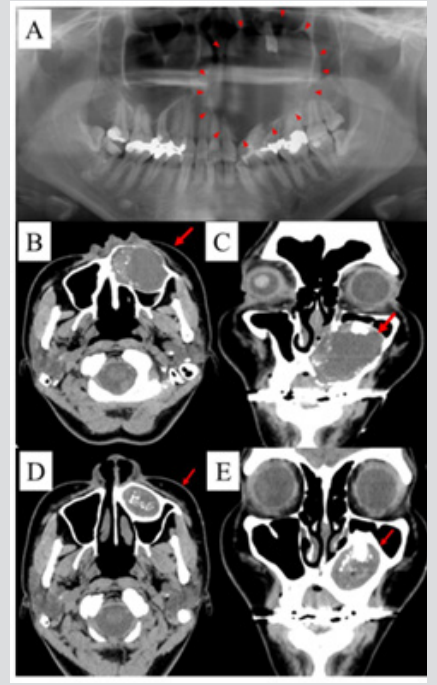

Figure 1: Panoramic $X$-ray and $C T$ at first visit and 6 months after fenestration.

The size of lesion on Panoramic radiograph (A) and CT (B,C) at first visit was approximately $40 \times 40 \times 36 \mathrm{~mm}$ and Many radiopacities were found within the radiolucent lesion, including calcified material and impacted tooth. Changes in the lesion were monitored by follow-up $\mathrm{CT}(\mathrm{D}, \mathrm{E})$, and a reduction in the tumor size with accompanying bone renewal were confirmed about 6 months after the fenestration procedure.

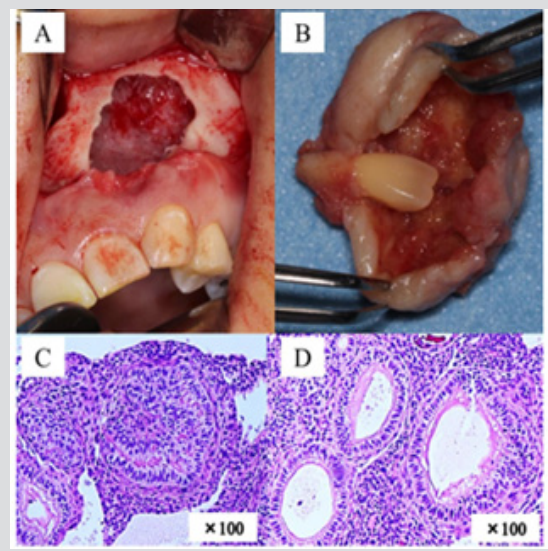

Figure 2: Intraoperative views, enucleated tumor and histological image.

We performed enucleation of the maxillary tumor and apicoectomy of the left upper canine under general anesthesia (A). The total size of the enucleated tissue, including the impacted tooth, was about $35 \mathrm{~mm}$ and had a thick cyst-like coating (B). The histopathological report of the enucleated lesion confirmed the diagnosis of follicular-type AOT. Ova-1 and polygon-shaped cells were present with ductal and corolla-like structures (C,D). 


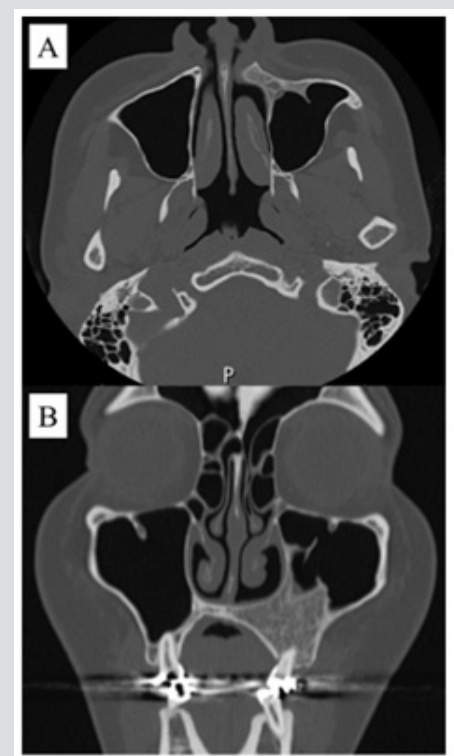

Figure 3: CT findings at 1 years after surgery.

At 1 year after the final surgery, the left cheek swelling, and the facial asymmetry had greatly improved. Bone renewal was also confirmed and the deformity of the bone of the front wall of the maxillary sinus and the alveolar bone of the buccal side had improved.

\section{Discussion}

AOT was first described in 1905 by Steensland. In 1907, Dreibladt called this lesion a pseudo-adenoameloblastoma, while in 1915, Harbitz proposed the term adamantoma; however, it was not until 1948 that Stafne [1] considered the lesion to be a distinct entity. Although it has since been thought of as a subtype of ameloblastoma, in 1969 Philipsen et al. coined the term "adenomatoid odontogenic tumor" that was adopted by the World Health Organization (WHO) classification for odontogenic tumors in 1971 [6]. It has been reported that AOTs account for $2 \%-7 \%$ of all odontogenic tumors [4-8]. They are more common in the maxilla than in the mandible (a ratio of 2:1), and they also occur more frequently in females $(64 \%-65 \%)$ and between the ages of 10 and 29 years $(74 \%-81 \%)$ compared with other groups [7,8]. Philipsen $[2,4]$ also reported that the male to female ratio was $6: 5$, nearly all cases occurred during the second decade of life (age 8 to 39 years), and most cases occurred in patients of Asian origin.

The typical presentation is with a maxillary swelling that can be either painless or painful. AOT is a slow-growing benign tumor that is not prone to recurrence. It has intraosseous and peripheral forms, with the former being differentiated into follicular and extrafollicular types based on radiographic findings [9]. Philipsen reported that the follicular, extrafollicular, and peripheral types occurred in $70.9 \%, 26.7 \%$, and $2.3 \%$ of cases, respectively $[2,4]$. AOTs usually measure $10-30 \mathrm{~mm}$, but they may grow to as large as $70 \mathrm{~mm}$ [10]. Horino [9] reported that the average size was 27.6 $\mathrm{mm}$, but that the average of those that developed into the maxillary sinus was only $41.6 \mathrm{~mm}$. Our maxillary case was consistent with this average. As mentioned, AOTs are usually solid, but may occasionally be cystic; indeed, only 11 cases have been described that arise in association with a dentigerous cyst [4]. That said, AOT has previously been described as an adenomatoid odontogenic cyst based on its form and histology [2]. Our case is therefore consistent with these alternative reports.

AOT often occurs in anterior areas, and about $70 \%$ of all cases are associated with an impacted tooth. The maxillary canines account for $41.7 \%$ of these cases, with all four canines involved in $60.1 \%$ of cases [4-10]. Therefore, it is perhaps unsurprising that $77 \%$ of follicular-type AOTs are initially diagnosed as dentigerous cysts [7]. Although the first premolar, third molar, second molar, and lateral incisor have been reported to be involved, the maxillary canine is the most often involved tooth. Interestingly, Philipsen reported that only one casehad a well-defined radiopaque mass, finding that all other lesions appeared as circumscribed unilocular radiolucent lesions around an unerupted tooth. Our patient was a young Asian woman with an AOT associated with an unerupted maxillary lateral incisor that was displacing the maxillary canine. Philipsen et al. [2] postulated that follicular-type AOT develops from cell nests within the dental lamina that surround the tooth. Furthermore, they suggest that the extrafollicular variants form from the epithelial nests situated at the borders of the conduit of tooth eruption. Consequently, the tooth would not be embedded by the developing AOT during eruption. As noted in the macroscopic observations, the tumor in the current case had a tissue tag that was attached to the epithelial lining in the bony depression.

Microscopic examination revealed that the tissue tag belonged to the epithelial lining of the dentigerous cyst. This raised three possibilities. First, the dentigerous cyst with the unerupted lateral 
incisor may have developed initially, and through a stimulus that is yet to be determined, the AOT arose from the epithelial nests in the dental lamina within the odontogenic cystic lining. Second, the findings reflect two different pathologies that were occurring concurrently in the lesion. Third, this may have been an entirely new variant. However, AOT has been reported to derive from a calcifying epithelial odontogenic cyst [2]. AOT is usually encapsulated and has few recurrences, allowing definitive treatment by surgical enucleation and curettage at presentation. However, our case was relatively large, and the maxillary deformity was a concern for the enucleation surgery. At biopsy, the tumor had a cyst-like structure with liquid or jelly-like material, so we initially performed fenestration to reduce the tumor size and improve the facial deformity. As a result, we could perform the enucleation with minimal surgical invasion, and satisfactory clinical results could be obtained without leaving unsatisfactory facial deformity. In cases such as ours, where the AOT has a cystic component and the patient has facial deformity, we advocate performing enucleation surgery after reducing the tumor size by fenestration surgery.

\section{References}

1. Stafne EC (1948) Epithelial tumors associated with developmental cysts of the maxilla: Report of 3 cases. Oral Surg 1(10): 887-894.

2. Philipsen HP, Khongkhunthiang P, Reichart PA (2016) The adenomatoid odontogenic tumor: an update of selected issues. J Oral Pathol Med 45(6): 394-398.

\section{ISSN: 2574-1241}

DOI: 10.26717/BJSTR.2019.15.002719

Hironobu Kobashi. Biomed J Sci \& Tech Res

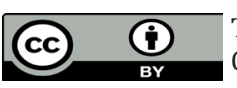

This work is licensed under Creative Commons Attribution 4.0 License

Submission Link: https://biomedres.us/submit-manuscript.php
3. Krishnamurthy K, Balaji RS, Devadiga S, Prasad RG (2014) Adenomatoid odontogenic tumor in the maxillary antrum: a rare case entity. J Pharm Bioallied Sci 6(5): 196-199.

4. Philipsen HP, Reichart PA, Zhang KH, Nikai H, Yu QX (1991) Adenomatoid odontogenic tumor: biologic profile based on 499 cases. J Oral Pathol Med 20(4): 149-158.

5. Vera Sempere FJ, Artes Martínez MJ, Vera Sirera B, Bonet Marco J (2006) Follicular adenomatoid odontogenic tumor: immunohistochemical study. Med Oral Patol Oral Cir Bucal 11(4): 305-308.

6. Philipsen HP, Birn H (1969) The adenomatoid odontogenic tumor. Ameloblastic adenomatoid tumor or adeno-ameloblastoma. Acta Pathol Microbiol Scand 75(3): 375-398.

7. Swasdison S, Dhanuthai K, Jainkittivong A, Philipsen HP (2008) Adenomatoid odontogenic tumors: an analysis of 67 cases in a Thai population. Oral Surg Oral Med Oral Pathol Oral Radiol Endod 105(2): 210-215.

8. Mohamed A, Singh AS, Raubenheimer EJ, Bouckaert MM (2010) Adenomatoid odontogenic tumor: review of the literature and an analysis of 33 cases from South Africa. Int J Oral Maxillofac Surg 39(9): 843-846.

9. Horino, K., Ohnishi, M (1996) Adenomatoid odontogenic tumor occupying maxillary sinus: a case report with histopathologic study and review of the literature. Niigata Dent J 26: 75.

10. Dhirawani RB, Pathak S, Mallikaarjuna K, Sharma A (2016) An adenomatoid odontogenic tumor in disguise. J Indian Soc Pedod Prev Dent 34(3): 291-293.

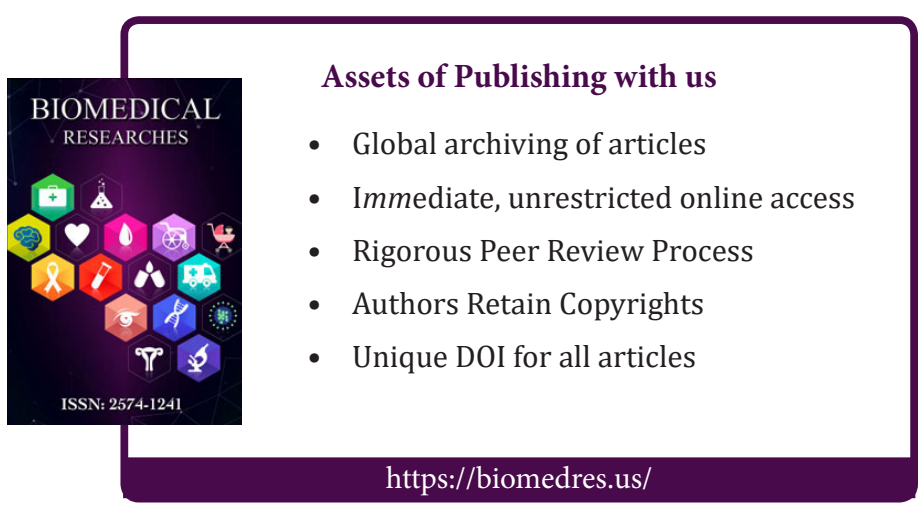

